\title{
FOLATE DEFICIENCY, PORPHYRIA, AND SEIZURES
}

A 12 year old, learning-disabled boy with epilepsy was admitted to the University of Connecticut Health Center, Farmington, CT because of symptomatic folate deficiency and newly diagnosed acute intermittent porphyria. Seizures and global developmental delays were noted by 12 months of age. Seizures required multiple drug combinations, and optimal control was obtained with carbamazepine, valproate, and methsuximide. The patient was hospitalized because of abdominal pain, vomiting, anorexia, and behavioral changes. He developed hypertension, hyponatremia, and anemia. The urine became brown in color, positive for urobilinogen, and showed elevated coproporphyrins, uroporphyrins, aminolevulinic acid, and porphobilinogen (PBG). Erythrocyte and maternal PBG deaminase measurements were decreased. $\mathrm{RBC}$ folate levels were deficient. Oral folate, $0.5 \mathrm{mg} / \mathrm{d}$, resulted in correction of the anemia and no recurrence of porphyric crises. (DiMario FJ $\mathrm{Jr}$ et al. Folate deficiency and acute intermittent porphyria in a 12-year-old boy. Neurology July 1993; 43: 1438-1439). (Reprints: Dr Francis J DiMario Jr, Pediatric Neurology, Bldg 12, UCONN Health Center, Farmington, CT 06030).

COMMENT. Serum folate should be determined in patients with acute intermittent porphyria requiring chronic anticonvulsant therapy. A folate deficiency induced by the AEDs may further reduce red blood cell production and precipitate a porphyric crisis. Folate supplements are beneficial as an adjunct to the carbohydrate loading in therapy for a porphyric crisis or its prevention.

\section{ANTIEPILEPTIC DRUGS}

\section{CARBAMAZEPINE-INDUCED SKIN RASH}

Of 335 children who were treated with carbamazepine at the Department of Paediatrics, Toyama Medical University, Japan, 33 (10\%) developed a skin rash. Five had exfoliative dermatitis and 2 had Stevens-Johnson syndrome. The eleventh day of therapy was the most frequent day of onset; $88 \%$ developed the rash between 8 and 15 days. Children over 6 years of age were more susceptible to skin rash than younger patients, with frequencies of $20 \%$ and $5 \%$, respectively. Leucopenia in 9 patients (27\%), thrombocytopenia in 6 (18\%), and hepatic dysfunction manifested by elevated transaminases in 9 (27\%) occurred concomitantly with the skin rash. (Konishi $\mathrm{T}$ et al. Carbamazepine-induced skin rash in children with epilepsy. Eur I Pediatr July 1993; 152: 605-608). (Respond: Dr T Konishi, Dept of Paediatrics, Toyama Medical and Pharmaceutical University, 2630, Sugitani, Toyama City, Japan). 
COMMENT. The frequency of skin rash with carbamazepine in this study is disturbingly high, particularly in children over 6 years of age. For additional reports, see Ped Neur Briefs Feb 1991, The introduction of dyes in the carbamazepine tablet in recent years may explain an apparent increase in reports of allergic skin reactions.

In a report from The Liverpool Hospital, New South Wales, Australia, the substitution of oxcarbazepine, a keto derivative thought to be less toxic than CBZ, caused a cross-reactive skin eruption in 3 adult patients who had previously developed exfoliative dermatitis with CBZ. (Beran RG. Epilepsia Jan/Feb 1993; 34: 163).

\section{$\underline{\text { STATUS EPILEPTICUS }}$}

\section{SERUM CORTISOL AND PROGNOSIS OF STATUS EPILEPTICUS}

The relation of serum cortisol and CSF $B$-endorphin levels to the prognosis of status epilepticus was evaluated in 27 adults treated in the Comprehensive Epilepsy Program of the Medical College of Virginia,

Richmond, VA. Poor prognosis was defined as death or severe neurologic or medical complications during or within one week after status epilepticus of more than 30 minutes. Blood cortisol levels were significantly elevated in patients with a poor outcome. CSF $B$-endorphins were significantly increased but did not correlate with prognosis. (Calabrese VP et al. Serum cortisol and cerebrospinal fluid $B$-endorphins in status epilepticus. Their possible relation to prognosis. Arch Neurol July 1993; 50: 689-693). (Reprints: Dr Calabrese, Dept of Neurology, MCV Station-Box 599, Richmond, VA 23298).

COMMENT. Serum cortisol determinations within 12 hours after control of status epilepticus may predict prognosis. The authors also propose that the rise in cortisol levels may contribute to the pathology of status epilepticus.

\section{OCCULT CORTICAL DYSPLASIA AND FOCAL STATUS}

Four patients, ages $4,6,13$, and 21 years, with life-threatening focal motor status epilepticus, normal pre-operative MRI, and focal cortical dysplasia defined only in the surgical specimens obtained by corticectomies, are reported from the Montreal Neurological Institute; Austin Hospital, Heidelberg, Australia; University of California, San Francisco; and Royal Children's Hospital, Melbourne, Australia. Seizures began between 3 months and 18 years of age, and therapy-resistant focal status developed after 18 months to 3 years, resulting in persistent motor deficits. A total of 12 MRIs failed to reveal abnormalities. Ictal SPECT was positive in 2 patients. Seizures were controlled after surgery, and 2 patients were seizure-free. (Desbiens R et al. Life-threatening focal status epilepticus due to occult cortical dysplasia. 